\title{
Intervenção Nutricional em Tempos de Pandemia por COVID-19
}

\section{Nutritional Intervention During COVID-19 Pandemic}

\author{
Mónica Pitta Grós Dias ${ }^{1}$, Carla Correia², Ana Catarina Moreira ${ }^{3}$
}

PALAVRAS-CHAVE: Avaliação Nutricional; COVID-19; Estado Nutricional; Inflamação; Pandemia; Terapia Nutricional

KEYWORDS: COVID-19; Inflammation; Nutrition Assessment; Nutrition Therapy; Nutritional Status; Pandemics

A pandemia de 2020 da COVID-19 provocada pela grave síndrome respiratória aguda coronavírus 2 (SARS-CoV-2) envolveu a maior parte do mundo, afetando mais de 180 países. Infelizmente, os mais vulneráveis e imunodeprimidos parecem ser mais suscetíveis a complicações graves de COVID-19. ${ }^{1}$

Existem vários fatores de risco para a infeção grave por COVID-19, como a degradação do estado nutricional e a presença de doenças crónicas não transmissíveis (DCNT), diabetes mellitus, doença pulmonar obstrutiva crónica, doença cardiovascular, HTA, obesidade e outras patologias que comprometem o estado de imunidade. Estas doenças são caracterizadas por estado de infla- mação sistémico, uma característica comum, que pode afetar a resposta dos doentes contra a COVID-19.1

O estado nutricional, padrão alimentar e estilo de vida são considerados fatores importantes pois podem desempenhar um papel positivo ou negativo na resposta à infeção por COVID-19. ${ }^{1}$

Até o momento, as estratégias de mitigação mais eficazes implementadas globalmente consistiram em incentivar boas práticas comuns de saúde pública, como lavar as mãos regularmente com sabão, medidas para prevenção da disseminação do vírus pela via área (usar máscara facial ${ }^{2}$ e tossir para o antebraço ${ }^{3}$ ), e distanciamento

1. Centro da Criança e do Adolescente - Hospital CUF Descobertas, Unidade de Nutrição do Hospital Dona Estefânia CHULC, Lisboa, Portugal. 2. Unidade de Nutrição do Hospital Dona Estefânia CHULC, Lisboa, Portugal. 3. Escola Superior de Tecnologia da Saúde de Lisboa - Instituto Politécnico de Lisboa; H\&TRC- Centro de Investigação em Saúde e Tecnologia, Lisboa, Portugal. 
social. Sabendo que um bom estado nutricional pode apoiar a função imunológica e prevenir o aparecimento de infeções graves ${ }^{4-7}$ levanta a questão, se manter um estado nutricional saudável poderá preparar o sistema imunitário de indivíduos não infetados com COVID-19.

A ligação entre a gravidade da infeção viral e as DCNT foi observada em outras infeções virais, como a gripe. ${ }^{8}$ A inflamação crónica e não tratada, muitas vezes subclínica, está implicada no aparecimento, progressão e desenvolvimento de DCNT.9,10 Acredita-se, igualmente, que a inflamação sistémica subjacente possa exacerbar a infeção por COVID-19.11

Uma estratégia eficaz para reduzir o risco de desenvolver DCNT é controlar as atividades mediadoras inflamatórias, pelos fatores de risco modificáveis, como dieta, exercício físico e escolhas de estilo de vida saudável.9,12 Apenas com a adoção de um padrão alimentar nutricionalmente equilibrado, consistente e a longo prazo, é possível avaliar os benefícios para a saúde humana. Enquanto a adoção de uma dieta e estilos de vida não saudáveis estão associados à subinflamação e ao aumento do stress oxidativo, o que poderá levar ao desenvolvimento de DCNT.9,13 Existem evidências consideráveis de que padrão alimentar, e até os nutrientes que consumimos afetam o funcionamento do sistema imunitário. 6,14,15

Alguns autores ${ }^{16,17}$ sugerem que um maior índice de massa corporal (IMC) ou excesso de adiposidade aumentam fatores de risco para complicações decorrentes da infeção por COVID-19. Possivelmente porque a população obesa tem maior prevalência de patologias pulmonares. ${ }^{18}$ Doentes com obesidade e comorbidades que comprometam a função cardíaca ou pulmonar têm maior risco de desenvolver doenças graves associadas à COVID-19, ${ }^{19}$ assim como os não obesos com estes fatores de risco. É recomendado manter um peso e uma composição corporal dentro das recomendações para estatura e sexo. ${ }^{19}$

O estado nutricional pode ter um impacto significativo na saúde geral de um indivíduo, na redução de DCNT e na suscetibilidade reduzida do desenvolvimento de infeções. No entanto, deve-se considerar que até ao momento não existem intervenções nutricionais baseadas em evidência ou estratégias de tratamentos disponíveis para prevenir a incidência ou gravidade da infeção por COVID-19. Da mesma forma, também não existe um único alimento ou medicamento natural, que tenha sido comprovado para prevenir a infeção por COVID-19, o que já foi esclarecido pela Organização Mundial da Saúde (OMS). ${ }^{17}$ No entanto, revendo estudos anteriores em relação a outras infeções virais, fica claro que o es- tado nutricional desempenha um papel significativo na resposta dos doentes. ${ }^{20}$ A presença de comorbidades nos doentes com COVID-19 é atualmente uma preocupação, ${ }^{16}$ questionando-nos se o estado nutricional desses doentes é fator de risco. Da mesma forma, para indivíduos que não foram afetados, adotar um padrão alimentar com propriedades anti-inflamatórias pode potencialmente prevenir ou minimizar infeções graves, em doentes com comorbidades que contraiam COVID-19. Em geral, é importante que a população siga uma dieta saudável. Existem padrões de dietas e nutrientes que potencialmente conferem propriedades anti-inflamatórias e imunomoduladoras. ${ }^{21-24}$

É particularmente importante neste momento monitorizar a população idosa, considerando que esta população não apresenta apenas predisposição para DCNT, mas também são vulneráveis ao risco aumentado de desnutrição, infeções e COVID-19.17 A idade em si é um fator de risco para o desenvolvimento de COVID-19, ${ }^{25}$ devido a um declínio funcional do sistema imunitário ao longo da vida. ${ }^{26,27}$ A desnutrição pode ocorrer por várias razões, incluindo más condições socioeconómicas, status mental, status social e uma série de outras questões multifatoriais. ${ }^{28}$ Frequentemente, existem deficiências nutricionais de cálcio, vitamina C, vitamina D, folato e zinco entre populações idosas ou carenciadas. ${ }^{29}$

A desnutrição pode deprimir o sistema imunológico já comprometido nos idosos, tornando-os suscetíveis a infeções. Uma dieta saudável e equilibrada pode oferecer os macros e micronutrientes, prebióticos, probióticos e simbióticos necessários, que podem contribuir para restaurar ou manter a função das células imunes aumentando assim a proteção contra as DCNT relacionadas à inflamação crónica. ${ }^{30}$

Vários padrões alimentares têm sido associados ao risco de condições inflamatórias e doenças respiratórias. Por outro lado, a dieta mediterrânea, caracterizada por uma ingestão relativamente alta em frutas, hortícolas, azeite, grãos integrais, frutos oleaginosos e gordura monoinsaturada pouco processada, produtos lácteos fermentados, peixe, aves, consumo moderado de vinho e baixo consumo de carnes processadas e vermelhas. ${ }^{9,30}$ Uma dieta equilibrada, com estes alimentos é rica em compostos anti-inflamatórios e imunomoduladores, incluindo vitaminas (C, D e E) e minerais (zinco, selénio, cobre, cálcio, iodo), que melhoram o estado nutricional. ${ }^{31}$

Alimentos associados à dieta mediterrânea e outros padrões alimentares saudáveis contêm compostos bioativos que vão além das vitaminas e minerais, incluindo compostos fenólicos bioativos e péptidos com potentes 
propriedades anti-inflamatórias, antitrombóticas e antioxidantes. Essas moléculas podem agir sinergicamente para prevenir e proteger contra manifestações inflamatórias. 9,31,32

Portanto, padrões alimentares saudáveis, como a dieta mediterrânea ou similar, são benéficos contra DCNT, mas, potencialmente, também podem conferir proteção contra infeções como a COVID-19, devido aos seus efeitos na melhoria do sistema imunitário.9,33,34

\section{SUPORTE NUTRICIONAL DE DOENTES COVID-19 INTERNADOS}

A terapia nutricional deve fazer parte integrante da abordagem terapêutica de doentes internados com COVID-19, nomeadamente os que apresentem maior gravidade, e doentes críticos..$^{35-37}$

O suporte nutricional é um dos componentes essenciais da prestação de cuidados de saúde a todos os doentes internados na Unidade de Infecciologia e Unidade de Cuidados Intensivos (áreas dedicadas a doentes COVID-19), com o objetivo de reduzir o risco de complicações. $^{35-37}$

A importância do estado nutricional no prognóstico do doente crítico tem sido cada vez mais reconhecida, estando a desnutrição associada a maior mortalidade, maior duração de hospitalização, aumento da dependência de ventilação mecânica e maior número de complicações infecciosas. ${ }^{37,38}$

No contexto da pandemia COVID-19, apesar da maioria dos doentes infetados com SARS-CoV-2 apresentar doença ligeira (cerca de 80\%), estima-se que entre 6\% a $10 \%$ dos doentes infetados tenham necessidade de hospitalização, em particular em Unidades de Cuidados Intensivos (UCls). ${ }^{38}$

Nos doentes em regime ambulatório sugere-se que possam beneficiar de acompanhamento nutricional, no sentido de adequar a ingestão alimentar ao sexo, idade, composição corporal, nível de atividade física e situação clínica se existente

A terapêutica nutricional para doentes com COVID-19 deve seguir as recentes recomendações da ESPEN e ASPEN. ${ }^{38,39}$ Em doentes com menor gravidade, com bom estado nutricional, deve-se assegurar a manutenção da composição corporal, prevenindo a perda de massa magra e assegurando o estado de hidratação.

\section{DOENTES EM ESTADO GRAVE OU CRÍTICO}

Os doentes em estado grave ou crítico apresentam alto risco de desnutrição, devendo ser estabelecidas as necessidades nutricionais, tipo de suporte a implementar, via de acesso, produto/fórmula nutricional, esquema de administração e de monitorização. Se a alimentação oral for insuficiente, inicialmente deve recorrer-se à utilização de suplementos nutricionais orais, ou se necessário considerar-se o suporte nutricional artificial (entérico ou parentérico). Se houver necessidade de restrição hídrica devem ser ponderadas fórmulas com maior densidade energética (> $1 \mathrm{kcal} / \mathrm{mL}$ ). A posição de decúbito dorsal, frequente na intervenção realizada nestes doentes, não condiciona a administração do suporte entérico. Apesar de ser objetivo final fornecer $25-30 \mathrm{kcal} / \mathrm{kg}$ de peso corporal, o início deve ser feito de forma gradual para evitar a síndrome de realimentação, iniciando-se com $15-20 \mathrm{kcal} / \mathrm{kg} / \mathrm{dia}$ e progredindo. O aporte proteico deve assegurar 1,2 a 2,0 g/kg peso corporal. ${ }^{38,39}$

\section{CONCLUSÃO}

Não existem terapêuticas nutricionais específicas comprovadas para prevenir a infeção por COVID-19, no entanto a literatura suporta a importância do estado nutricional na resposta dos doentes a infeções virais. No doente infetado devem ser privilegiadas as intervenções nutricionais para a manutenção da composição corporal, prevenindo a perda de massa magra. No doente crítico deve ser ponderado o suporte nutricional artificial para atingir as necessidades nutricionais.

\section{RESPONSABILIDADES ÉTICAS}

CONFLITOS DE INTERESSE: Os autores declaram não possuir conflitos de interesse.

SUPORTE FINANCEIRO: O presente trabalho não foi suportado por nenhum subsídio ou bolsa.

PROVENIÊNCIA E REVISÃO POR PARES: Não comissionado; revisão externa por pares.

\section{ETHICAL DISCLOSURES}

CONFLICTS OF INTEREST: The authors have no conflicts of interest to declare.

FINANCING SUPPORT: This work has not received any contribution, grant or scholarship.

PROVENANCE AND PEER REVIEW: Not commissioned; externally peer reviewed. 


\section{REFERÊNCIAS}

1. Zabetakis I, Lordan R, Norton C, Tsoupras A. COVID-19: the inflammation link and the role of nutrition in potential mitigation. Nutrients. 2020;12:E1466. doi:10.3390/nu12051466.

2. Cheng VC, Wong SC, Chuang VW, So SY, Chen JH, Sridhar S, et al. The role of community-wide wearing of face mask for control of coronavirus disease 2019 (COVID-19) epidemic due to SARS-CoV-2. J Infect. 2020 (in press) doi: 10.1016/j. jinf.2020.04.024.

3. Centers for Disease Control and Prevention. How to Protect Yourself \& Others. [accessed on 1 April 2020] Available from: https://www.cdc.gov/coronavirus/2019-ncov/prevent-getting-sick/prevention.html.

4. Wu D, Lewis ED, Pae M, Meydani SN. Nutritional modulation of immune function: analysis of evidence, mechanisms, and clinical relevance. Front Immunol. 2019:9:3160. doi: 10.3389/ fimmu.2018.03160.

5. Grant WB, Lahore H, McDonnell SL, Baggerly CA, French CB, Aliano JL, et al. Evidence that Vitamin D Supplementation Could Reduce Risk of Influenza and COVID-19 Infections and Deaths. Nutrients. 2020;12:988. doi:10.3390/nu12040988.

6. Childs CE, Calder PC, Miles EA. Diet and Immune Function. Nutrients. 2019:11:1933. doi: 10.3390/nu11081933.

7. Calder PC, Carr AC, Gombart AF, Eggersdorfer M. Optimal Nutritional Status for a Well-Functioning Immune System Is an Important Factor to Protect against Viral Infections. Nutrients. 2020;12:1181. doi: 10.3390/nu12041181.

8. Van Kerkhove MD, Vandemaele KA, Shinde V, Jaramillo-Gutierrez G, Koukounari A, Donnelly CA, et al. Risk factors for severe outcomes following 2009 influenza A (H1N1) infection: a global pooled analysis. PLoS Med. 2011;8:e1001053. doi:10.1371/journal.pmed.1001053.

9. Tsoupras A, Lordan R, Zabetakis I. Inflammation, not cholesterol, is a cause of chronic disease. Nutrients. 2018, 10, 604.

10. Hotamisligil GS. Inflammation, metaflammation and immunometabolic disorders. Nature. 2017; 542:177-85.

11. Yang J, Zheng Y, Gou X, Pu K, Chen Z, Guo Q, et al. Prevalence of comorbidities in the novel wuhan coronavirus (COVID-19) infection: A systematic review and meta-analysis. Int J Infect Dis. 2020;94:91-5.

12. Yu E, Malik VS, Hu FB. Cardiovascular disease prevention by diet modification: JACC health promotion series. J Am Coll Cardiol. 2018;72:914-26.

13. Wu G. Important roles of dietary taurine, creatine, carnosine, anserine and 4-hydroxyproline in human nutrition and health. Amino Acids. 2020;52:329-60.

14. Chandra RK. Nutrition, immunity and infection: From basic knowledge of dietary manipulation of immune responses to practical application of ameliorating suffering and improving survival. Proc Natl Acad Sci USA. 1996;93:14304-7.

15. Hulsewé KW, van Acker BA, von Meyenfeldt MF, Soeters PB. Nutritional depletion and dietary manipulation: Effects on the immune response. World J Surg. 1999;23:536-44.

16. World Health Organization. COVID-19 and NCDs. [accessed on 1 April 2020] Available from: https://www.who.int/internal-publications-detail/covid-19-and-ncds.

17. Centers for Disease Control and Prevention. Morbidity and Mortality Weekly Report (mmwr): Preliminary Estimates of the Prevalence of Selected Underlying Health Conditions among Patients with Coronavirus Disease 2019-February 12-March 28. 2020. accessed on 1 April 2020] Available from: https://www.cdc.gov/mmwr/volumes/69/wr/mm6913e2.htm?s_cid=mm6913e2_w.
18. Costa D, Barbalho MC, Miguel GP, Forti EM, Azevedo $J \mathrm{~L}$. The impact of obesity on pulmonary function in adult women. Clinics. 2008;63:719-24. doi: 10.1590/s180759322008000600002

19. Wu Z, McGoogan JM. Characteristics of and Important Lessons from the Coronavirus Disease 2019 (COVID-19) Outbreak in China: Summary of a Report of 72314 Cases from the Chinese Center for Disease Control and Prevention. JAMA.2020 (in press). doi: 10.1001/jama.2020.2648.

20. Beck MA, Handy J, Levander OA. Host nutritional status: the neglected virulence factor. Trends Microbiol. 2004;12:41723. doi:10.1016/j.tim.2004.07.007.

21. Georgousopoulou EN, Kouli GM, Panagiotakos DB, Kalogeropoulou A, Zana A, Chrysohoou C, et al. Anti-inflammatory diet and 10-year (2002-2012) cardiovascular disease incidence: The ATTICA study. Int J Cardiol. 2016;222:473-8. doi: 10.1016/j.ijcard.2016.08.007.

22. de Boer A, van de Worp WRPH. Hageman GJ, Bast A. The effect of dietary components on inflammatory lung diseases - a literature review. Int J Food Sci Nutr. 2017:68:771-87. doi:10 .1080/09637486.2017.1288199

23. Lago JH, Toledo-Arruda AC, Mernak M, Barrosa KH, Martins MA, Tibério IF, et al. Structure-activity association of flavonoids in lung diseases. Molecules. 2014;19:3570-95. doi: 10.3390/molecules19033570

24. Phillips CM, Chen LW, Heude B, Bernard JY, Harvey NC, Duiits L, et al. Dietary Inflammatory Index and Non-Communicable Disease Risk: A Narrative Review. Nutrients. 2019;11:1873. doi: 10.3390/nu11081873.

25. Wu C, Chen X, Cai Y, Xia J, Zhou X, Xu S, et al. Risk factors associated with acute respiratory distress syndrome and death in patients with coronavirus disease 2019 pneumonia in Wuhan, China. JAMA Intern Med. 2020 (in press). doi:10.1001/jamainternmed.2020.0994.

26. Pae M, Meydani SN, Wu D. The role of nutrition in enhancing immunity in aging. Aging Dis. 2012;3:91-129.

27. Meyer KC. The role of immunity and inflammation in lung senescence and susceptibility to infection in the elderly. Semin Respir Crit Care Med. 2010;31:561-74. doi:10.1055/s-0030-1265897.

28. Volkert D, Visser M, Corish CA, Geisler C, de Groot L, Cruz-Jentoft AJ, et al. Joint action malnutrition in the elderly (MaNuEL) knowledge hub: summary of project findings. Eur Geriatr Med. 2020;11:169-177. doi:10.1007/s41999-01900264-3.

29. Power SE, Jeffery IB, Ross RP, Stanton C, O'Toole PW, O'Connor EM, et al. Food and nutrient intake of Irish community-dwelling elderly subjects: who is at nutritional risk? J Nutr Health Aging. 2014;18:561-72. doi:10.1007/s12603-0140449-9.

30. Martinez-Gonzalez MA, Bes-Rastrollo M. Dietary patterns, Mediterranean diet, and cardiovascular disease [published correction appears in Curr Opin Lipidol. 2014;25:326]. Curr Opin Lipidol. 2014;25:20-6. doi:10.1097/ MOL.0000000000000044.

31. Casas R, Sacanella E, Estruch R. The immune protective effect of the Mediterranean diet against chronic low-grade inflammatory diseases. Endocr Metab Immune Disord Drug Targets. 2014:14:245-54. doi:10.2174/187153031466614 0922153350.

32. Cheng S, Tu M, Liu H, Zhao G, Du M. Food-derived antithrombotic peptides: Preparation, identification, and interactions with thrombin. Crit Rev Food Sci Nutr. 2019:59(sup1):S81-S95. doi: 10.1080/10408398.2018.1524363. 
33. Shah R, Makarem N, Emin M, Liao M, Jelic S, Aggarwal B. Mediterranean diet components are linked to greater endothelial function and lower inflammation in a pilot study of ethnically diverse women. Nutr Res. 2020;75:77-84. doi:10.1016/j.nutres.2020.01.004.

34. Muscogiuri G, Barrea L, Savastano S, Colao A. Nutritional recommendations for CoVID-19 quarantine. Eur J Clin Nutr. 2020 14:1-2. doi: 10.1038/s41430-020-0635-2.

35. First Affiliated Hospital, Zhejiang University School of Medicine. Handbook of COVID-19 Prevention and Treatment. [accessed on 1 April 2020] Available from: https://covid-19. alibabacloud.com/.

36. Thibault, R., Quilliot, D., Seguin, P., Tamion, F., Schneider, S., Déchelotte, P., Comité Educationnel et de Pratique Clinique de la SFNCM. Épidémie virale Covid-19 - Stratégie de prise en charge nutritionnelle à l'hôpital. Paris: Société Francophone Nutrition Clinique et Métabolisme; 2020.

37. Wang D, Hu B, Hu C, Zhu F, Liu X, Zhang J, et al. Clinical Characteristics of 138 Hospitalized Patients With 2019 Novel Coronavirus-Infected Pneumonia in Wuhan, China. JAMA 2020;323:1061-9. doi: 10.1001/jama.2020.1585.

38. Singer P, Blaser AR, Berger MM, Alhazzani W, Calder PC, Casaer MP, et al. ESPEN guideline on clinical nutrition in the intensive care unit. Clin Nutr. 2019;38:48-79. doi: 10.1016/j. clnu.2018.08.037.

39. Da Silva JSV, Seres DS, Sabino K, Adams SC, Berdahl GJ, Citty SW, et al. ASPEN Consensus Recommendations for Refeeding Syndrome. Nutr Clin Pract. 2020;35:178-95. 